\title{
A novel compound heterozygous mutation of $S L C 26 A 4$ in two Chinese families with nonsyndromic hearing loss and enlarged vestibular aqueducts
}

\author{
GUANG-JIE ZHU* , LU-SEN SHI*, HAN ZHOU, YE YANG, JIE CHEN and XIA GAO \\ Department of Otorhinolaryngology, Head and Neck Surgery, Drum Tower Hospital Affiliated \\ to Nanjing University Medical School, Nanjing, Jiangsu 210008, P.R. China
}

Received January 24, 2017; Accepted August 10, 2017

DOI: $10.3892 / \mathrm{mmr} .2017 .7690$

\begin{abstract}
Enlarged vestibular aqueduct (EVA)-associated hearing loss is frequently detected in individuals carrying the SLC26A4 mutation in the Chinese population. The present study aimed to identify the causative SLC26A4 coding mutations in a patient group with nonsyndromic hearing loss (NSHL) and EVA. Genomic DNA was extracted from blood samples obtained from 52 NSHL patients with EVA and from 60 normal controls. The mutation analysis for 20 coding exons of SLC26A4 was performed by direct sequencing. The results of the mutational analysis showed that there were two probands from two separate families suffering from bilateral sensorineural hearing loss with EVA, carrying the same novel compound heterozygous mutation of SLC26A4 (c.1644_1645insA and c.2168A>G). Other members of the two families had heterozygous mono-allelic mutations with normal hearing. However, neither of these mutations were detected in the 60 normal controls. These results are the first, to the best of our knowledge, to link the compound heterozygote mutation,
\end{abstract}

Correspondence to: Dr Jie Chen or Dr Xia Gao, Department of Otorhinolaryngology, Head and Neck Surgery, Drum Tower Hospital Affiliated to Nanjing University Medical School, 321 Zhongshan Road, Nanjing, Jiangsu 210008, P.R. China

E-mail: njjiechen@163.com

E-mail: gaoxiadth@outlook.com

${ }^{*}$ Contributed equally

Abbreviations: EVA, enlarged vestibular aqueduct; NSHL, nonsyndromic hearing loss; STAS, anti-sigma factor antagonist; SHL, sensorineural hearing loss; PS, Pendred syndrome; MD, Mondini dysplasia; PTA, pure-tone audiometry; CT, computed tomography; MRI, magnetic resonance imaging; ABR, auditory brainstem response; DPOAE, distortion product otoacoustic emissions; PCR, polymerase chain reaction; NCBI, National Center for Biotechnology Information; PKA, putative protein kinase A

Key words: nonsyndromic hearing loss, enlarged vestibular aqueduct, SLC26A4, novel compound heterozygous mutation
c.1644_1645insA and c.2168A $>$ G, in the SLC26A4 gene to NSHL patients with EVA. The two mutations identified in the present study were located in the anti-sigma factor antagonist domain, the core region for plasma membrane targeting of anion transporters, which suggested that the reduced or complete loss of SLC26A4 function was the direct cause of hearing loss in the two patients. These results provide a foundation for further elucidating the genetic factors responsible for EVA-associated NSHL.

\section{Introduction}

Enlarged vestibular aqueduct (EVA) syndrome, most frequently found by radiological examination in patients with nonsyndromic hearing loss (NSHL), is typically characterized by congenital, bilateral profound sensorineural hearing loss (SHL) or progressive hearing loss (HL) $(1,2)$. EVA in SHL is often accompanied with or without Pendred syndrome (PS; MIM: 274600), which is often caused by mutation of the pendrin protein encoded by the SLC26A4 gene. Autosomal recessive NSHL with $S L C 26 A 4$ mutations has been frequently reported to present with inner ear malformations, including EVA, which can be associated with Mondini dysplasia (MD), and abnormality of cochlear spirals detected using computed tomography (CT) and magnetic resonance imaging (MRI) (3-5). SLC26A4 comprises 21 exons and produces an $86 \mathrm{kDa}$ protein containing 780 amino acids. The SLC26A4 protein, a member of the solute carrier family $26 \mathrm{~A}$ and also known as pendrin, is mapped on chromosome 7q31 (6). Pendrin, the gene product of SLC26A4, is important in the regulation of endolymphatic $\mathrm{pH}$ and the maintenance of endocochlear potential by mediating the exchange of fluid and ions, including formate, bicarbonate, chloride and iodide ions (7). SLC26A4 mutation can cause PS and autosomal recessive nonsyndromic hearing impairment locus 4 (MIM: 600791)-type NSHL. To date, >200 SLC26A4 mutations have been reported in patients with nonsyndromic EVA or PS (http://www.healthcare.uiowa.edu/labs/pendredandbor/slcMutations.htm). In addition, $98.9 \%$ of the SLC26A4 mutants detected in patients with EVA are from the Chinese population (8). A previous study reported that children with PS and EVA showed improved performance in speech perception, compared with a reference group with an unknown 
cause of hearing impairment following cochlear implantation (9). Expanding the SLC26A4 mutation spectrum may be beneficial for molecular assessment, enabling early diagnosis and planning effective clinical strategies. Through the use of targeted sequence capture and direct sequencing, the present study investigated 20 coding exons of the SLC26A4 gene in 52 NSHL patients diagnosed with EVA by CT and MRI. The results provided the first evidence, to the best of our knowledge, of the compound heterozygous mutation p.R549Kfs*15 and p.H723R in SLC26A4, which expands on the SLC26A4 mutation spectrum in the Chinese population.

\section{Patients and methods}

Subjects and clinical investigations. All Chinese individuals enrolled in the present study were recruited from the Ear, Nose and Throat Department of Drum Tower Hospital Affiliated to Nanjing University (Nanjing, China). A total of 60 normal-hearing Chinese individuals underwent pure-tone audiometry (PTA) testing and otoscopy at the Drum Tower Hospital (male/female, 32/28; age, $7.86 \pm 4.71$ years). Those individuals with normal tympanum morphology and an average threshold of PTA $(250-8,000 \mathrm{~Hz})<25 \mathrm{~dB}$ HL were considered as normal-hearing controls. A total of 52 NSHL patients with EVA (male/female, 30/22; age, 3.52 \pm 6.13 years) and their parents underwent systematic clinical examinations and audiometric evaluations prior to the patients receiving a cochlear implant at Drum Tower Hospital. The average age of the 52 NSHL patients with EVA was significantly lower, compared with that of the normal controls, determined using Student's t-test $(\mathrm{P}<0.001)$. As the majority of the normal controls were inpatients suffering from adenoid hypertrophy and chronic tonsillitis, there were 37 pre-language HL patients in the group of 52 NSHL patients with EVA in the present study. In addition to PTA and otoscopy, the patients underwent tympanometry, auditory brainstem response (ABR), distortion products otoacoustic emissions (DPOAE) and imaging examinations. Imaging of the ear was performed by CT and MRI. The patients or their parents were also interviewed to obtain a detailed medical history and family history, details of the mother's health during pregnancy, the patient's clinical history, including history of head or brain injury, infection and the use of medicines, including aminoglycoside antibiotics. The data collected, including characteristics and age at diagnosis, are listed in Table I.

All participants and the parents of all minors recruited in the present study provided written informed consent, and the study protocol was performed in accordance with institutional bioethics guidelines approved by the Research and Ethics Committee of Drum Tower Hospital (201601502).

DNA isolation, polymerase chain reaction (PCR) and sequence data analyses. Blood samples were collected following the provision of written informed consent. Total genomic DNA was extracted from the peripheral blood using a gDNA Isolation Micro kit (Watson Biotechnologies, Shanghai, China). All 20 coding exons of the SLC26A4 gene were amplified using PCR with primers designed using Primer3 software (http://primer3.ut.ee/; Table II). The PCR mixture contained $250 \mu \mathrm{m}$ dNTP Mixture, 10X Ex Taq buffer and Ex Taq (5 U/ $\mu \mathrm{l})$
Table I. Clinicopathological characteristics of 52 patients with NSHL and 60 normal controls.

\begin{tabular}{|c|c|c|c|}
\hline Characteristic & $\begin{array}{l}\text { Patients with } \\
\text { NSHL and } \\
\text { EVA n }(\%)\end{array}$ & $\begin{array}{c}\text { Normal } \\
\text { controls n }(\%)\end{array}$ & P-value \\
\hline Age $^{\mathrm{a}}$ (years) & $3.52 \pm 6.13$ & $7.86 \pm 4.71$ & $<0.0001$ \\
\hline \multicolumn{4}{|l|}{ Sex } \\
\hline Male & $30(57.69)$ & $32(53.33)$ & \multirow[t]{2}{*}{0.644} \\
\hline Female & $22(42.31)$ & $28(46.67)$ & \\
\hline \multicolumn{4}{|l|}{ Family history } \\
\hline Yes & $14(26.92)$ & NA & \\
\hline No & $38(73.08)$ & NA & \\
\hline \multicolumn{4}{|l|}{ Hearing loss } \\
\hline Pre-language & $37(71.15)$ & NA & \\
\hline Post-language & $15(28.85)$ & NA & \\
\hline \multicolumn{4}{|l|}{$\begin{array}{l}\text { Modini } \\
\text { dysmorphia }\end{array}$} \\
\hline With & $2(3.85)$ & NA & \\
\hline Without & $50(96.15)$ & NA & \\
\hline \multicolumn{4}{|l|}{ c. $2168 \mathrm{~A}>\mathrm{G}$} \\
\hline Positive & $9(17.31)$ & 0 & \\
\hline Negative & 43 (82.69) & 60 & \\
\hline \multicolumn{4}{|l|}{ c.1644_1645insA } \\
\hline Positive & $2(3.85)$ & 0 & \\
\hline Negative & $50(96.15)$ & 60 & \\
\hline
\end{tabular}

${ }^{\mathrm{a} A g e}$ is presented as the mean \pm standard deviation. Difference between groups were determined using Student's t-test. Sex differences were analyzed using Pearson's $\chi^{2}$ test. NSHL, nonsyndromic hearing loss; EVA, enlarged vestibular aqueduct; NA, not applicable.

polymerase (Takara Biotechnology Co., Ltd., Dalian, China), and underwent 30 cycles of amplification with denaturation at $96^{\circ} \mathrm{C}$ for $20 \mathrm{sec}$, annealing at $62^{\circ} \mathrm{C}$ for $20 \mathrm{sec}$, extension at $72^{\circ} \mathrm{C}$ for $60 \mathrm{sec}$, and extension at $72^{\circ} \mathrm{C}$ for $5 \mathrm{~min}$ in a PTC-200 PCR thermal cycler (Bio-Rad Laboratories, Inc., Hercules, CA, USA). The PCR amplified products were purified and then sequenced using Sanger sequencing with an ABI 3730XL genetic analyzer (Beijing Genomics Institute, Beijing, China). The sequence data were analyzed by comparing them with the sequence of SLC26A4 (NM_000441.1) of the National Center for Biotechnology Information (NCBI) using DNAStar 5.0 software (DNAStar, Inc., Madison, WI, USA). In addition, the 60 normal controls were checked at the same locus.

Statistical analysis. Statistical analysis was performed using SPSS 22.0 for Windows (IBM SPSS, Armonk, NY, USA). The difference in age between the NSHL patients with EVA and the normal controls was determined using Student's t-test. The sex differences were analyzed using Pearson's $\chi^{2}$ test. The statistical associations of the prevalence of the novel compound heterozygous mutation of SLC26A4 were analyzed using Fisher's exact test. $\mathrm{P}<0.05$ was considered to indicate a statistically significant difference. 
Table II. Specific primers used to amplify the coding exons of SLC26A4.

\begin{tabular}{llll}
\hline Exon & \multicolumn{1}{c}{ Forward primer $\left(5^{\prime}-3^{\prime}\right)$} & \multicolumn{1}{c}{ Reverse primer (5'-3') } & Product size (bp) \\
\hline 2 & GGGGACTGGGTGGAACT & GCCCGAGACTGATGGAG & 678 \\
3 & GCAAATTGGTGTGACTGAG & GAAGGGTAAGCAACCATCTGTCAC & 294 \\
4 & GTTGGGCAAAATAATCTAACGCA & GCAGGCAAAACACTGAAATCC & 575 \\
5 & GGTCCGGCTCAGCTTCTT & GCACCTGACCTAAAACAACGT & 481 \\
6 & AGGAAGGGGAGTGATAGGGT & GTCTCAAACTCCTGGGCTCA & 497 \\
$7-8$ & CATGGTTTTCATGTGGGAAGATTC & AGACTGACTTACTGACTTAATGT & 502 \\
9 & GAGGACAAAGAAATCAGCCAGT & CCCCTTCTTTAGCTGACACC & 456 \\
10 & ATCAGGTGCTATTTCTTG & TTTCAGGTGAGGGAGTG & 526 \\
$11-12$ & TGTTCAGTTTTGGGCTTGAG & TCACATGGAAGACTTCATGGC & 663 \\
13 & TGTTTGTGGATCATTGA & GCACAGCAGTAGAGGAC & 527 \\
15 & ACCTTTCAGGGTTATGG & TTTCTCCCTTTGGCTAC & 491 \\
16 & TTGAGTGCTGCTACCC & TTCTCATTGCCCTACA & 346 \\
17 & CCCTTTGAGAAATAGCC & TTGCCAAGAAATACACT & 587 \\
19 & CTACCCACCATAGAAGG & GCAATACTGGACAACCC & 626 \\
21 & ATTTAGCACCTCCACG & CCACAGTCCCAGATAG & 375 \\
\hline
\end{tabular}

\section{Results}

Clinical and audiological evaluation. The novel compound heterozygous mutation, p.R549Kfs*15 and p.H723R, in SLC26A4 was detected in a 4-year-old boy (A3) from family A and a 5-year-old girl (B3) from family B in the present study (Fig. 1A-F). The two families were from different cities of the Jiangsu province of China, and there was no relationship between the families. There was no history of hearing loss in the families of the two the two individuals or any clinical evidence showing any syndrome or disease, for example hypothyroidism. There was no history of previous illness, including meningitis, or trauma, and pregnancies were normal. There were no occurrences of congenital NSHL or syndromic hearing infection, including PS, in either family. Audiological examinations were performed and the results of PTA test showed that the two probands (A3 and B3) exhibited bilateral profound SHL (Fig. 2). This type of hearing cannot be improved with ear hearing aids and they presented with deficiency in language development. The results of the ABR showed that no representative wave was initiated under the highest intensity (105 dB), and the DPOAE showed no representative response in the frequency range of $1-4 \mathrm{kHz}$ in the two probands. The results of the otoscopic examinations revealed a normal external auditory canal and tympanic membranes, and tympanometric results were normal. The two probands showed no vestibular symptoms and had a walking age of 12 months. Their thyroids were of normal size, and the results of the laboratory investigations showed normal blood parameters and thyroid function. The other members of the two families had normal hearing (Fig. 2).

Radiological examination. EVA was defined as a diameter at the midpoint between the common crus and the external aperture of $>1.5 \mathrm{~mm}$ (10). The temporal bone CT examinations of the two probands showed no cochlear malformations, however, bilateral EVA was present and the width of the vestibular aqueduct was $>1.5 \mathrm{~mm}$ (Fig. 3A and B). On MRI examination, enlargement of the endolymphatic sac and duct was observed in the left and right ears of the two probands (Fig. 3C-F).

Mutation analysis. Sequencing of the 20 SLC26A4 coding exons in the 52 patients with EVA revealed the same compound heterozygous mutation in the two probands, the 4-year-old boy and a 5-year-old girl, from the two separate families. The two variants comprised an insertion of an adenine (c.1644_1645insA) in exon 15 (Fig. 1C) and an adenine to guanine substitution (c.2168A $>$ G) in exon 19 (Fig. 1E). The first insertion was predicted to cause a frameshift and produce a truncated protein by a premature stop (p.R549Kfs*15). This variation has not been reported previously. Individuals A1 in family A, and B1 and B4 in family B were heterozygous for this variation. The second mutation led to the substitution of histidine by arginine (p.H723R). This variation was present in the NCBI dbSNP (rs121908362) and Deafness Variation Databases of the University of Uiowa (http://www.healthcare. uiowa.edu/labs/pendredandbor/slcMutations.htm).

The missense mutation identified in SLC26A4 has been previously reported to cause PS or NSHL with EVA $(11,12)$. Patient A2 in family A and B2 in family B were heterozygous for this variation. The affected members (A3 and B3) of the two families were heterozygous for the p.R549Kfs*15 and p.H723R mutations. None of the compound heterozygous mutations were detected in the 60 ethnically-matched normal control subjects or in the 50 remaining NSHL patients with EVA. This finding indicated that the p.R549Kfs*15 variation was the NSHL-causing mutation in the two families, as a compound heterozygous mutation with p.H723R. 
A

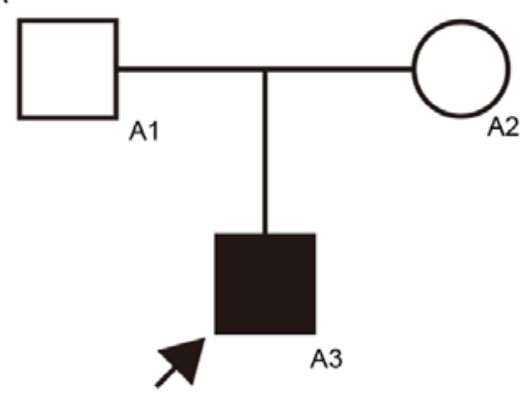

B

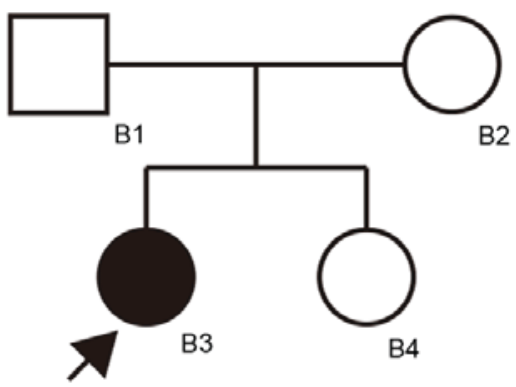

C

TTAGA TTT TCCAGTCCTATT TTCTAT $\frac{\text { TGGCAAT }}{\mathrm{R}} \frac{\mathrm{GTC}}{\mathrm{F}} \frac{\mathrm{GAT}}{\mathrm{S}} \frac{\mathrm{GGT}}{\mathrm{V}} \frac{\text { TT TAAAA }}{\mathrm{F}} \frac{\mathrm{K}}{\mathrm{K}}$

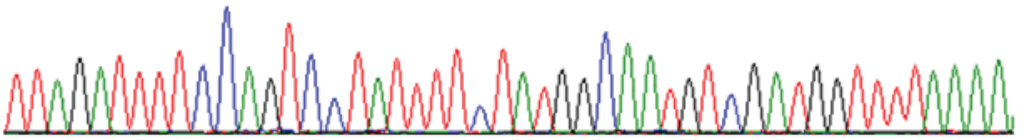

D

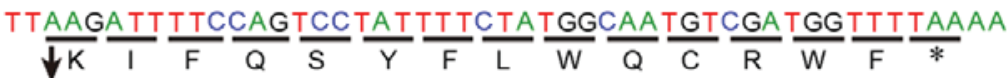

c. $1644 \_1645$ ins A
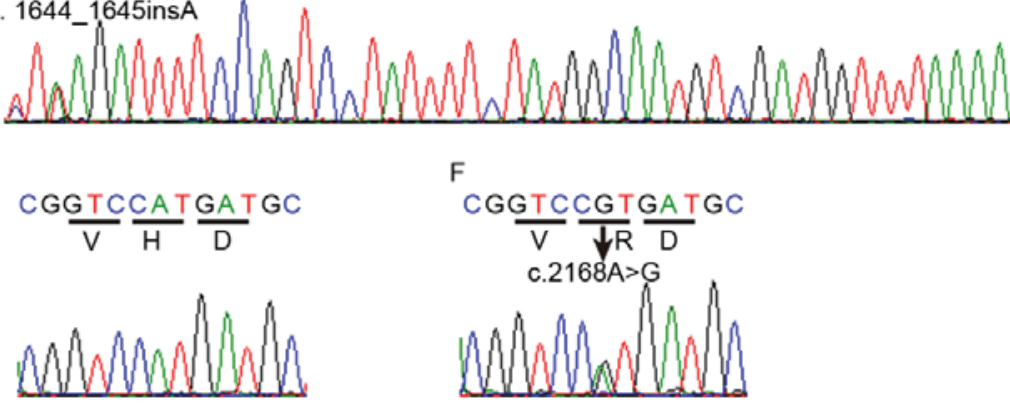

$\mathrm{F}$

CGGTC $\frac{C G T G A T G C}{\downarrow R}$

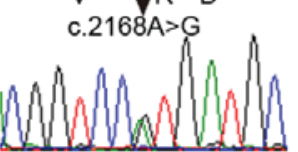

Figure 1. Autosomal recessive pedigrees of the two families. Pedigree showing the (A) 4-year-old boy (A3) from family A and (B) 5-year-old girl (B3) from family B with profound hearing loss examined in the present study (shaded and indicated with arrows) and the unaffected sister (2 years old) in family B. (C) Normal allele c.1642 and protein product of SLC26A4. (D) Heterozygous inheritance of the SLC26A4 insertion c.1644_1645insA (p.R549Kfs*15) was detected using Sanger sequencing in the two probands. (E) Normal allele c.2162 and protein product of SLC26A4. (F) c.2168A >G missense mutation (p.H723R, rs121908362) detected using Sanger sequencing in the two patients.
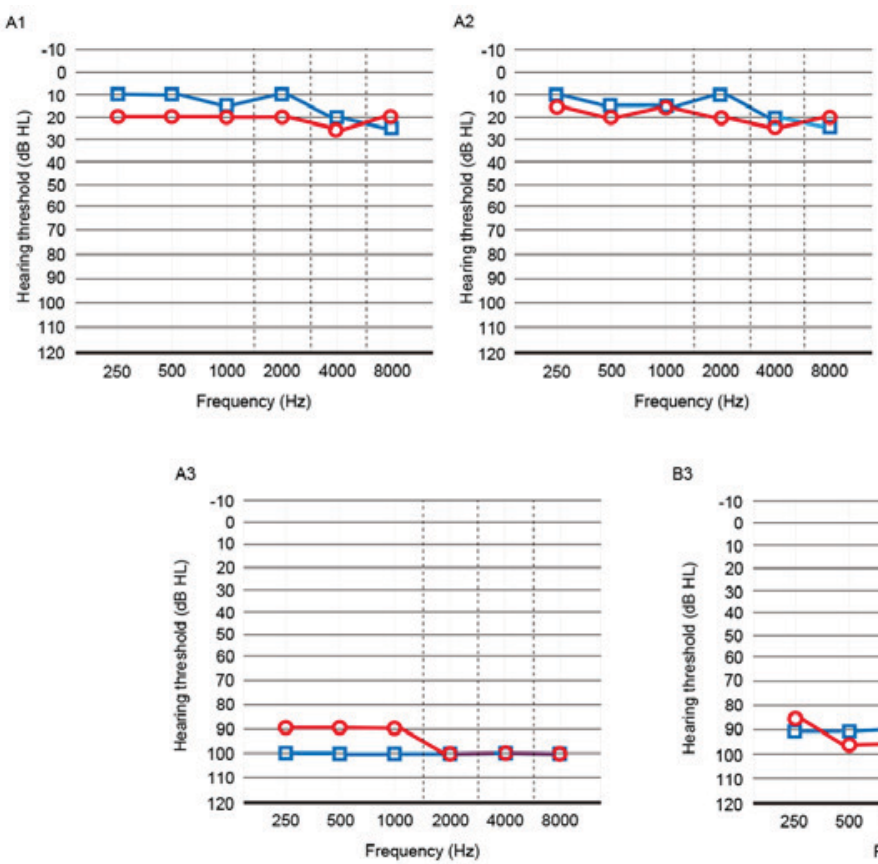

B1

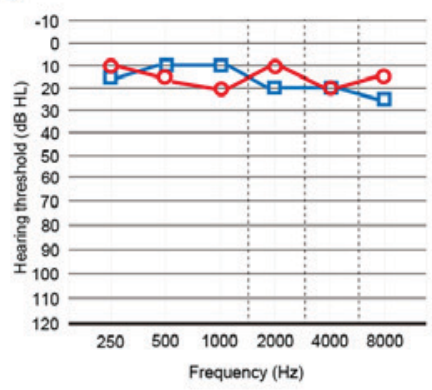

B2

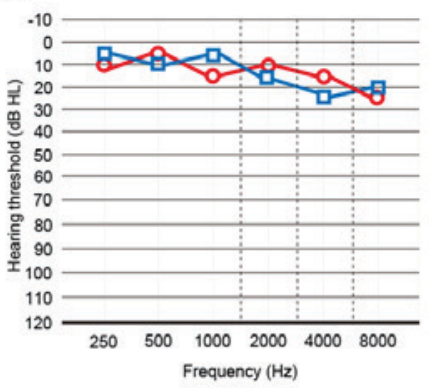

83

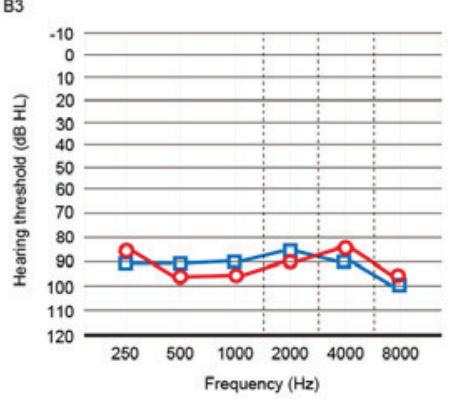

B4

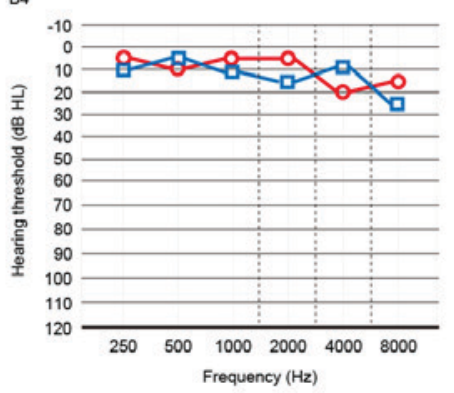

Figure 2. Bilateral pure tone audiograms from individuals in families A and B. Red circles in the audiograms represent right air conduction thresholds and blue squares represent left conduction thresholds. B4 was examined using behavior observation audiometry. 

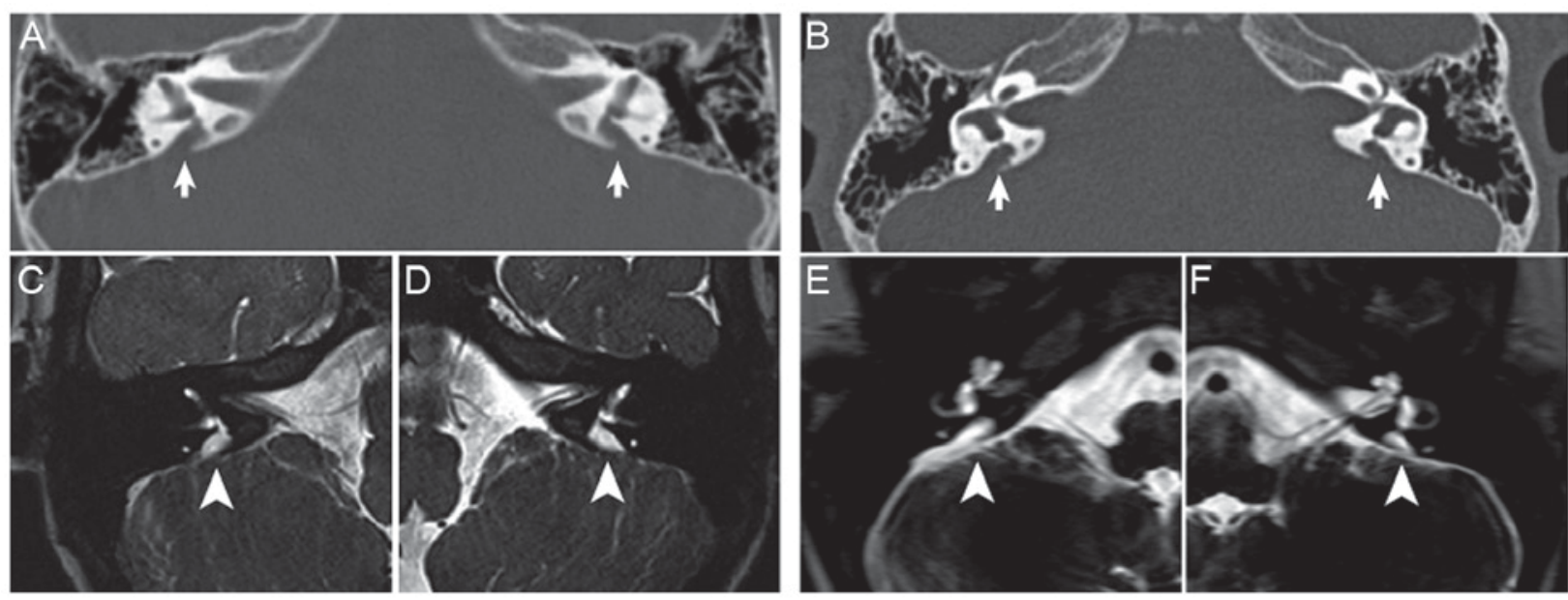

Figure 3. Radiological examinations. Axial temporal bone computed tomography images of patient (A) A3 and (B) B3 show enlarged vestibular aqueducts (white arrows). Inner ear magnetic resonance images of the (C) right and (D) left ear of patient A3, and (E) right and (F) left ear of patient B3 show the enlarged endolymph sac of the two probands (white arrows heads).

\section{Discussion}

In the present study, a novel compound heterozygous mutation of SLC26A4 (c.1644_1645insA, c.2168A>G) was identified in two patients with profound bilateral SHL and EVA. The parents of the two patients and an unaffected member in family B (B4) carried a mutant allele separately with a normal allele and had normal hearing. The compound heterozygous mutation was inherited from the mutant alleles of the father and mother in the two probands. Therefore, it was concluded that the two affected families reported were consistent with an autosomal recessive disorder caused by bi-allelic function loss of the pendrin protein. Compound heterozygous patients with two SLC26A4 mutant alleles have been reported in up to $50 \%$ of Chinese patients with HL and exhibit significant genetic heterogeneity in Asian populations (13-15). The mutant allele of c.2168A>G (p. $\mathrm{H} 723 \mathrm{R}$ ) has been reported to be a common pathogenic mutation among Chinese, Korean and Japanese patients with HL (14-17). The incidence of this mutation in patients with NSHL and EVA in the present study was $9 / 52$ (17.31\%; 95\% CI, 8.23-30.33\%). It has been reported that the mutant pendrin comprised p.H723R is localized predominantly in endoplasmic reticulum and was found to lack the activity of anion exchange (18). It was also found that patients with two bi-allelic mutations of p.H723R or a compound heterozygous mutation with the other mutation often suffered from bilateral SHL (19-21).

The other mutant allele c.1644_1645insA mutation of SLC26A4 was identified as a novel compound heterozygous mutation with p.H723R in the present study. The incidence of this mutation in patients with NSHL and EVA in the present study was $2 / 52$ (3.85\%; 95\% CI, 0.47-13.21\%). The predicted result of the c.1644_1645insA mutation was a frameshift beginning at codon 549 (Arg to Lys) and a premature translation stop at position 562 in exon 15 . The different conserved amino acid change (p.H723R) in exon 19 of pendrin caused by mutation of c. $2168 \mathrm{~A}>\mathrm{G}$ and the early translational termination in exon 15 at the coding region of carboxyl terminus caused by c.1644_1645insA led to a deleterious effect on protein function, eventually resulting in a pathologic phenotype with profound HL and EVA.
Pendrin, as with other proteins in the SLC26 family, carries a sulfate transporter and anti-sigma factor antagonist (STAS) domain in its carboxy-terminus. Aravind and Koonin (22) reported that the STAS domain encompassed amino acids 535-573 and 654-729 of pendrin. The p.R549Kfs*15 and p.H723R mutations identified in the two probands in the present study were involved in the STAS domain. The STAS domain in proteins of the SLC26 family is reported to be involved in nucleotide binding and interactions with other proteins $(23,24)$. The function of the STAS domain can be affected or lost by mutations reported in a number of patients with PS or EVA, which indicates that the STAS domain may be an important intracellular component of pendrin in the function of plasma membrane targeting and retention. The majority of the mutations in the STAS domain lead to its reduced or complete loss of function as an anion transporter, including iodide efflux $(18,25-27)$.

It has also been confirmed that the c.2168A $>\mathrm{G}$ (p.H723R) mutant is a hot-spot region of the SLC26A4 mutation in Chinese patients with PS and EVA $(12,16)$, also affecting the putative protein kinase A (PKA) binding site. The PKA and STAS domains located in the carboxyl terminus region of pendrin are considered to be important components in the targeting of proteins to the plasma membrane (28).

In addition, the c.1644_1645insA (p.R549Kfs*15) mutation identified in the present study can cause an early stop codon and truncated protein. The early stop codon causes nonsense-mediated mRNA decay and these mRNAs are rapidly decayed (29). As the c.2168A $>\mathrm{G}$ (p.H723R) mutant is on another allele, the patients reported had no pendrin proteins with a normal function and exhibited NSHL with EVA. A number of reasons indicate the novel compound heterozygous mutation of the SLC26A4 gene, including p.R549Kfs" 15 and p.H723R, as the pathologic mutation. The 20 coding exon sequencing analysis of SLC26A4 in the 52 NSHL patients with EVA revealed the compound heterozygous mutation (c.1644_1645ins A, c.2168A $>$ G) in two individuals $(2 / 52$; $3.85 \%)$. The high prevalence of this novel compound heterozygous mutation of SLC26A4 was almost $4 \%$ among the patients with NSHL and EVA in the present study $(\mathrm{P}=0.03$ with Fisher's 
exact test). Therefore, the novel compound heterozygous mutation of SLC26A4 identified in the present study expands on the wide mutational spectrum of SLC26A4. The results of the present study provide a foundation for future investigations of the molecular mechanisms of SLC26A4 mutations associated with HL and EVA.

\section{Acknowledgements}

The present study was supported by the General Program from Natural Science Foundation of China to Dr Gao Xia (grant nos. 81371090 and 81570921 ). The authors would like to thank the Core Medical and Genetics Laboratory of Drum Tower Hospital Affiliated to Nanjing University Medical School.

\section{References}

1. Usami S, Abe S, Weston MD, Shinkawa H, Van Camp G and Kimberling WJ: Non-syndromic hearing loss associated with enlarged vestibular aqueduct is caused by PDS mutations. Hum Genet 104: 188-192, 1999.

2. Pique LM, Brennan ML, Davidson CJ, Schaefer F, Greinwald J Jr and Schrijver I: Mutation analysis of the SLC26A4, FOXI1 and KCNJ10 genes in individuals with congenital hearing loss. Peer J 2: e384, 2014.

3. Reardon W, OMahoney CF, Trembath R, Jan H and Phelps PD: Enlarged vestibular aqueduct: A radiological marker of pendred syndrome, and mutation of the PDS gene. QJM 93: 99-104, 2000.

4. Campbell C, Cucci RA, Prasad S, Green GE, Edeal JB, Galer CE, Karniski LP, Sheffield VC and Smith RJ: Pendred syndrome, DFNB4, and PDS/SLC26A4 identification of eight novel mutations and possible genotype-phenotype correlations. Hum Mutat 17: 403-411, 2001.

5. Pryor SP, Madeo AC, Reynolds JC, Sarlis NJ, Arnos KS, Nance WE, Yang Y, Zalewski CK, Brewer CC, Butman JA and Griffith AJ: SLC26A4/PDS genotype-phenotype correlation in hearing loss with enlargement of the vestibular aqueduct (EVA): Evidence that Pendred syndrome and non-syndromic EVA are distinct clinical and genetic entities. J Med Genet 42: 159-165, 2005.

6. Everett LA, Glaser B, Beck JC, Idol JR, Buchs A, Heyman M, Adawi F, Hazani E, Nassir E, Baxevanis AD, et al: Pendred syndrome is caused by mutations in a putative sulphate transporter gene (PDS). Nat Genet 17: 411-422, 1997.

7. Kopp P, Pesce L and Solis-S JC: Pendred syndrome and iodide transport in the thyroid. Trends Endocrinol Metab 19: 260-268, 2008 .

8. Chai Y, Huang Z, Tao Z, Li X, Li L, Li Y, Wu H and Yang T: Molecular etiology of hearing impairment associated with nonsyndromic enlarged vestibular aqueduct in East China. Am J Med Genet A 161A: 2226-2233, 2013.

9. Van Nierop JW, Huinck WJ, Pennings RJ, Admiraal RJ, Mylanus EA and Kunst HP: Patients with pendred syndrome: Is cochlear implantation beneficial?. Clin Otolaryngol 41: 386-394, 2016.

10. Cremers CW, Admiraal RJ, Huygen PL, Bolder C, Everett LA, Joosten FB, Green ED, van Camp G and Otten BJ: Progressive hearing loss, hypoplasia of the cochlea and widened vestibular aqueducts are very common features in Pendred's syndrome. Int J Pediatr Otorhinolaryngol 45: 113-123, 1998.

11. Coyle B, Reardon W, Herbrick JA, Tsui LC, Gausden E, Lee J, Coffey R, Grueters A, Grossman4 A, Phelps PD, et al: Molecular analysis of the PDS gene in Pendred syndrome. Hum Mol Genet 7: 1105-1112, 1998.

12. Reyes S, Wang G, Ouyang X, Han B, Du LL, Yuan HJ, Yan D, Dai P and Liu XZ: Mutation analysis of SLC26A4 in mainland Chinese patients with enlarged vestibular aqueduct. Otolaryngol Head Neck Surg 141: 502-508, 2009.
13. Peng Q, Huang S, Liang Y, Ma K, Li S, Yang L, Li W, Ma Q, Liu Q, Zhong $\mathrm{B}$ and Lu X: Concurrent genetic and standard screening for hearing impairment in 9317 southern chinese newborns. Genet Test Mol Biomarkers 20: 603-608, 2016.

14. Tsukamoto K, Suzuki H, Harada D, Namba A, Abe S and Usami S: Distribution and frequencies of PDS (SLC26A4) mutations in Pendred syndrome and nonsyndromic hearing loss associated with enlarged vestibular aqueduct: A unique spectrum of mutations in Japanese. Eur J Hum Genet 11: 916-922, 2003.

15. Shin JW, Lee SC, Lee HK and Park HJ: Genetic screening of GJB2 and SLC26A4 in korean cochlear implantees: Experience of soree ear clinic. Clin Exp Otorhinolaryngol 5 (Suppl 1): S10-S13, 2012.

16. Wang QJ, Zhao YL, Rao SQ, Guo YF, Yuan H, Zong L, Guan J, Xu BC, Wang DY, Han MK, et al: A distinct spectrum of SLC26A4 mutations in patients with enlarged vestibular aqueduct in China. Clin Genet 72: 245-254, 2007.

17. Wu CC, Yeh TH, Chen PJ and Hsu CJ: Prevalent SLC26A4 mutations in patients with enlarged vestibular aqueduct and/or Mondini dysplasia: A unique spectrum of mutations in Taiwan, including a frequent founder mutation. Laryngoscope 115: 1060-1064, 2005.

18. Yoon JS, Park HJ, Yoo SY, Namkung W, Jo MJ, Koo SK, Park HY, Lee WS, Kim KH and Lee MG: Heterogeneity in the processing defect of SLC26A4 mutants. J Med Genet 45: 411-419, 2008.

19. Pang X, Chai Y, Chen P, He L, Wang X, Wu H and Yang T: Mono-allelic mutations of SLC26A4 is over-presented in deaf patients with non-syndromic enlarged vestibular aqueduct. Int J Pediatr Otorhinolaryngol 79: 1351-1353, 2015.

20. Sagong B, Baek JI, Lee KY and Kim UK: A novel frameshift mutation of SLC26A4 in a korean family with nonsyndromic hearing loss and enlarged vestibular aqueduct. Clin Exp Otorhinolaryngol 10: $50-55,2017$

21. Yao J, Qian X, Bao J, Wei Q, Lu Y, Zheng H, Cao X and Xing G: Probing the effect of two heterozygous mutations in codon 723 of SLC26A4 on deafness phenotype based on molecular dynamics simulations. Sci Rep 5: 10831, 2015.

22. Aravind L and Koonin EV: The STAS domain-a link between anion transporters and antisigma-factor antagonists. Curr Biol 10: R53-R55, 2000.

23. Sharma AK, Ye L, Baer CE, Shanmugasundaram K, Alber T, Alper SL and Rigby AC: Solution structure of the guanine nucleotide-binding STAS domain of SLC26-related SulP protein Rv1739c from Mycobacterium tuberculosis. J Biol Chem 286: 8534-8544, 2011.

24. Ko SB, Zeng W, Dorwart MR, Luo X, Kim KH, Millen L, Goto H, Naruse S, Soyombo A, Thomas PJ and Muallem S: Gating of CFTR by the STAS domain of SLC26 transporters. Nat Cell Biol 6: 343-350, 2004.

25. Taylor JP, Metcalfe RA, Watson PF, Weetman AP and Trembath RC: Mutations of the PDS gene, encoding pendrin, are associated with protein mislocalization and loss of iodide efflux: Implications for thyroid dysfunction in Pendred syndrome. J Clin Endocrinol Metab 87: 1778-1784, 2002.

26. Pera A, Dossena S, Rodighiero S, Gandía M, Bottà G, Meyer G, Moreno F, Nofziger C, Hernández-Chico C and Paulmichl M: Functional assessment of allelic variants in the SLC26A4 gene involved in Pendred syndrome and nonsyndromic EVA. Proc Natl Acad Sci USA 105: 18608-18613, 2008.

27. Scott DA, Wang R, Kreman TM, Andrews M, McDonald JM, Bishop JR, Smith RJ, Karniski LP and Sheffield VC: Functional differences of the PDS gene product are associated with phenotypic variation in patients with Pendred syndrome and non-syndromic hearing loss (DFNB4). Hum Mol Genet 9: 1709-1715, 2000.

28. Bizhanova A, Chew TL, Khuon S and Kopp P: Analysis of cellular localization and function of carboxy-terminal mutants of pendrin. Cell Physiol Biochem 28: 423-434, 2011.

29. Frischmeyer PA and Dietz HC: Nonsense-mediated mRNA decay in health and disease. Hum Mol Genet 8: 1893-1900, 1999. 